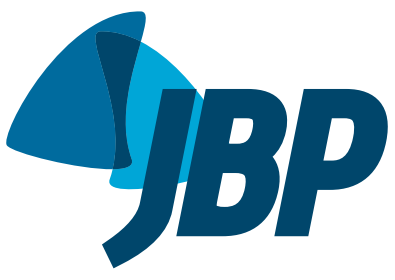

\title{
Use of thrombolytic agents in the treatment of acute pulmonary thromboembolism: things are not as simple as you might think
}

\author{
Carlos Henrique Miranda',a
}

I congratulate Fernandes et al. on their review article entitled "Reperfusion in Acute Pulmonary Thromboembolism" (PTE) published in the JBP. ${ }^{(1)}$

I would like to make some practical comments on and comparisons with the use of thrombolytic agents in acute myocardial infarction (MI), initially with regard to high-risk group patients who present with hemodynamic instability-systolic blood pressure (SBP) $<90 \mathrm{mmHg}$ or a sustained $\geq 40-\mathrm{mmHg}$ decrease in SBP for 15 minutes in the absence of other reasons, such as newonset arrhythmias, hypovolemia, or sepsis. ${ }^{(2)}$ The use of thrombolytic agents in high-risk group patients has been reported to reduce the relative risk of death by $80 \%$; however, according to one study, up to two thirds of such patients do not receive fibrinolytic agents. ${ }^{(3)}$ This reduction in the relative risk of death is substantially larger than that observed in acute ST-segment elevation MI (STEMI), with ranges from $20-30 \%$, according to one study. ${ }^{(4)}$ Nevertheless, thrombolytic agents are widely used in acute STEMI, and not prescribing fibrinolytic therapy, especially when primary angioplasty is unavailable, is considered poor medical practice. I believe that the use of thrombolysis in high-risk PTE should be encouraged, provided that absolute contraindications are taken into account. Recently, a directive published in the Brazilian Official Federal Government Journal ${ }^{(5)}$ mandated that the indication for alteplase in treating PTE be incorporated into the Brazilian Unified Health Care System. We should also be alert to the fact that several patients have borderline SBP $(90-110 \mathrm{mmHg})$, and sometimes it is difficult to establish baseline blood pressure levels in order to determine whether there is a $\geq 40-\mathrm{mmHg}$ decrease. In such cases, I recommend measuring serum lactate levels. Serum lactate levels $>2.0 \mathrm{mg} / \mathrm{dL}$ suggest that the observed blood pressure level is not adequate for tissue perfusion. If that is the case, the patient also meets the criteria for circulatory shock, since lactate levels are essential to detecting hemodynamic collapse. Some investigations have shown that lactate in this context is an independent predictor of mortality, being a better prognosticator than either troponin or $\mathrm{N}$-terminal prohormone of brain natriuretic peptide (NT-proBNP). ${ }^{(6,7)}$ Such a patient also meets criteria for thrombolysis.

With regard to the use of thrombolytic agents in intermediate-risk patients, I believe that the controversy continues, because a single randomized study does not provide enough evidence for a final verdict. We should consider that the markers used have a low positive predictive value for identifying patients at high risk for complications. Optimal cut-off values for troponin and NT-proBNP have not yet been established and may vary according to the method used for determining their levels. With regard to echocardiography, there is no methodological standardization for evaluating the right ventricle. Therefore, I consider it a great challenge to establish new parameters with adequate accuracy for selecting patients who are truly at risk, and perhaps these are the patients who can benefit from fibrinolytic therapy.

One study ${ }^{(8)}$ that investigated thrombolysis with tenecteplase in intermediate-risk patients had numerous setbacks. First, the thrombolytic agent chosen. In this setting, the largest experience has been with alteplase. Tenecteplase's ease of administration as an i.v. bolus is a plus; however, the disadvantage is its duration of action (40 min), whereas alteplase's duration of action is 2 hours, which guarantees longer exposure time for thrombus dissolution, especially in patients with high thrombotic load. In a meta-analysis of the use of thrombolytic agents in PTE, alteplase did not increase the risk of bleeding (OR $=1.07 ; 95 \% \mathrm{CI}$ : 0.43-2.62); however, this risk increased considerably with the use of tenecteplase (OR $=5.02$; 95\% CI: 2.72-9.26). ${ }^{(9)}$ Therefore, I believe that alteplase should be considered the first choice for reperfusion in PTE. Second, the study by Meyer et al. ${ }^{(8)}$ did not adjust the dose of tenecteplase for elderly patients, and although the use of tenecteplase was found to reduce the composite outcome of mortality and hemodynamic decompensation from $5.6 \%$ to $2.6 \%(p=0.02)$, it caused a higher number of intracranial bleeds ( $2.0 \%$ vs. $0.2 \% ; p=0.003)$. In that study, ${ }^{\left({ }^{8}\right)}$ subgroup analysis demonstrated that the clinical benefit was limited to patients $\leq 75$ years of age (OR = 0.33 ; $95 \% \mathrm{CI}: 0.13-0.85)$. Of the 11 patients who had intracranial hemorrhage, $9(82 \%)$ were $\geq 75$ years of age. ${ }^{(8)}$ Armstrong et al. ${ }^{(10)}$ addressed thrombolysis with tenecteplase followed by angiography in the treatment of acute STEMI, initially demonstrating an increase in the rates of intracranial bleeding in patients $\geq 75$ years of age who received tenecteplase $(1.0 \%$ vs. $0.2 \%$; $p=$ $0.04)$; after an amendment to the study protocol, with the dose of tenecteplase being reduced by half in patients $\geq 75$ years of age, the bleeding rate was equivalent between groups $(0.5 \%$ vs. $0.3 \% ; p=0.45)$. Third, the recommended dose of unfractionated heparin for use with the thrombolytic agent in PTE is an i.v. bolus of $80 \mathrm{IU} /$ $\mathrm{kg}$, followed by $18 \mathrm{IU} / \mathrm{kg}$ per hour continuous infusion, with adjustment of the activated partial thromboplastin time (aPTT) to 2.0-2.5 $\times$ normal. One study on the use of fibrinolytic agents in acute STEMI showed a reduction in bleeding with the use of a lower-dose heparin regimen 
(60 IU/kg bolus; maximum, $4000 \mathrm{IU}$; followed by 12 $\mathrm{IU} / \mathrm{kg}$ per hour continuous infusion; maximum, 1000 $\mathrm{IU} / \mathrm{h}$ ) with a target aPTT between 1.5 and 2.5. ${ }^{(11)}$ After thrombolysis for PTE, it is not uncommon that patients are started on heparin therapy and that the first aPTTs reveal non-coagulability of blood, precisely at this stage when the risk of bleeding is more critical. In the study by Meyer et al., ${ }^{(8)} 30 \%$ of the patients had heparin levels above recommended levels. Why not start infusion more cautiously and gradually adjust it upward if necessary? And, finally, that study reported low mortality $(2.4 \%$ in the tenecteplase group vs. $3.2 \%$ in the control group), which may be due to early diagnosis and treatment in European centers, from where the patients were recruited. ${ }^{(8)}$ However, a sample of patients in Brazil had a higher mortality rate of approximately $20 \%$, which may be due to delayed diagnosis because of difficulties within the Brazilian Unified Health Care System. ${ }^{(12)}$ In this context, the role of fibrinolytic agents may be more prominent.

PTE is a neglected health problem, especially when compared with acute STEMI. With regard to treatment of low-risk patients, the institution of heparin therapy is sufficient. For high-risk group patients, we need to promote the use of thrombolysis. And, regarding intermediate-risk group patients, we still need to improve our scientific basis before establishing definitive approaches.

\section{REFERENCES}

1. Fernandes CJCDS, Jardim CVP, Alves JL Jr, Oleas FAG, Morinaga LTK, Souza R. Reperfusion in acute pulmonary thromboembolism. J Bras Pneumol. 2018;44(3):237-243. https://doi.org/10.1590/s1806 37562017000000204

2. Konstantinides SV, Torbicki A, Agnelli G, Danchin N, Fitzmaurice D, Galie N, et al. 2014 ESC guidelines on the diagnosis and management of acute pulmonary embolism. Eur Heart J. 2014;35(43):3033-69, 3069a-3069k.

3. Stein PD, Matta F. Thrombolytic therapy in unstable patients with acute pulmonary embolism: saves lives but underused. Am J Med. 2012;125(5):465-70. https://doi.org/10.1016/j.amjmed.2011.10.015

4. Verstraete M. Thrombolytic treatment in acute myocardial infarction Circulation. 1990;82(3 Suppl):II96-109

5. Brasil. Ministério da Saúde. Secretaria de Ciência, Tecnologia e Insumos Estratégicos. Portaria No. 37 (2018 Sep 11); Diário Oficial da União. 176(1):203.

6. Vanni S, Socci F, Pepe G, Nazerian P, Viviani G, Baioni M, et al. High plasma lactate levels are associated with increased risk of in-hospital mortality in patients with pulmonary embolism. Acad Emerg Med. 2011;18(8):830-5. https://doi.org/10.1111/j.1553-2712.2011.01128.x

7. Vanni S, Viviani G, Baioni M, Pepe G, Nazerian P, Socci F, et al Prognostic value of plasma lactate levels among patients with acute

pulmonary embolism: the thrombo-embolism lactate outcome study. Ann Emerg Med. 2013;61(3):330-8. https://doi.org/10.1016/j. annemergmed.2012.10.022

8. Meyer G, Vicaut E, Danays T, Agnelli G, Becattini C, BeyerWestendorf J, et al. Fibrinolysis for patients with intermediate-risk pulmonary embolism. N Engl J Med. 2014;370(15):1402-11. https:// doi.org/10.1056/NEJMoa1302097

9. Marti C, John G, Konstantinides S, Combescure C, Sanchez O, Lankeit $M$, et al. Systemic thrombolytic therapy for acute pulmonary embolism: a systematic review and meta-analysis. Eur Heart $\mathrm{J}$. 2015;36(10):605-14. https://doi.org/10.1093/eurheartj/ehu218

10. Armstrong PW, Gershlick AH, Goldstein P, Wilcox R, Danays T, Lambert $\mathrm{Y}$, et al. Fibrinolysis or primary $\mathrm{PCl}$ in $\mathrm{ST}$-segment elevation myocardial infarction. N Engl J Med. 2013;368(15):1379-87. https:// doi.org/10.1056/NEJMoa1301092

11. Giugliano RP, McCabe $\mathrm{CH}$, Antman EM, Cannon CP, Van de Werf $F$, Wilcox RG, et al. Lower-dose heparin with fibrinolysis is associated with lower rates of intracranial hemorrhage. Am Heart $\mathrm{J}$ 2001;141(5):742-50. https://doi.org/10.1067/mhj.2001.114975

12. Volschan A, Albuquerque DC, Tura BR, Knibel Mde F, Souza PC Toscano ML. Pulmonary embolism: multicenter registry in tertiary hospitals. Rev Bras Ter Intensiva. 2009;21(3):237-46. 


\title{
Authors' reply
}

\section{Reflections on the use of thrombolytic agents in acute pulmonary embolism}

\author{
Caio Julio Cesar dos Santos Fernandes 1,2,a, Carlos Vianna Poyares Jardim, ${ }^{1, b}$, José Leonidas Alves Jr', ${ }^{1,2, c}$, \\ Francisca Alexandra Gavilanes Oleas ${ }^{1, d}$, Luciana Tamie Kato Morinaga ${ }^{1, e}$, Rogério de Souza ${ }^{1, f}$
}

The use of systemic thrombolytic agents for the treatment of acute pulmonary embolism is a controversial subject, in which evidence and belief eventually clash. While there is no dispute regarding the benefits of the procedure for high-risk patients, ${ }^{(1)}$ this indication is much more debatable for intermediate-high-risk patients. These latter patients are characterized by maintaining adequate levels of tissue perfusion at the expense of right ventricle injury. It is quite tempting to imagine that pharmacological intervention at this point would prevent progression to right ventricular failure, cardiovascular collapse, and death. It is also intuitive to seek a long-term benefit from the use of thrombolysis in intermediate-high-risk patients; after all, by reducing the thrombotic load, it would be possible to reduce any residual vascular obstruction, thereby reducing the risk of chronic thromboembolic pulmonary hypertension. However, thrombolysis indisputably increases the risk of bleeding. So what to do? The physiological rationale is not always the best path to take. In such cases, seeking the best evidence available can provide better answers.

The study by Meyer et al.(2) is the largest and best study to date to evaluate thrombolysis versus heparin therapy in intermediate-risk patients systematically, although it is not the only one. (1) The number of patients evaluated in the study by Meyer et al.(2) is larger than the total number of patients in all studies that investigated alteplase, the most traditionally used drug in such cases (1006 vs. 657). This results in tenecteplase being the most commonly investigated thrombolytic agent in phase III trials in pulmonary embolism today. In addition, because of the large number of patients, the study by Meyer et al.(2) has an $80 \%$ power of detection of intergroup differences. All those studies, with the one by Meyer et al. (2) being the most representative, tend to converge on the same finding: while the use of thrombolytic agents poses an increased risk of bleeding, which is greater in the population known to be at risk, such as the elderly, the benefits of thrombolysis, whether with alteplase, tenecteplase, urokinase, or streptokinase, appear to be quite modest. Traditional heparin therapy appears to be quite safe, with a mortality rate of $1.8 \%$ if good medical practices are followed. Monitoring of intermediate-high-risk intensive care patients and prompt institution of reperfusion at the first sign of hemodynamic instability are mandatory prerequisites. However, if these prerequisites are met, and with such low mortality rates, is it worth performing thrombolysis, since conventional therapy is effective? The most reasonable solution appears to be conventional therapy, intensive monitoring, and early reperfusion if there is any sign of hemodynamic instability. And, as suggested earlier, it is possible that lactate levels play a role in this monitoring.

Long-term benefits also do not justify the use of thrombolysis. Data from a study by Konstantinides et al. ${ }^{(3)}$ identified no benefits in mortality rates, residual dyspnea, or diagnosis of chronic thromboembolic pulmonary hypertension. If the short-term benefit is small, the medium-term benefit is zero, and there is the risk of further morbidity, such as bleeding, why do it indiscriminately? Of course, if the choice if for thrombolysis, hemorrhage should be prevented by dose adjustment for weight and age, pressure control, and use of a proton pump inhibitor. Even so, does the benefit justify the risk? To date, the best available evidence tells us that it does not.

\section{REFERENCES}

1. Fernandes CJCDS, Jardim CVP, Alves JL Jr, Oleas FAG, Morinaga LTK, Souza R. Reperfusion in acute pulmonary thromboembolism. J Bras Pneumol. 2018;44(3):237-243. https://doi.org/10.1590/s180637562017000000204

2. Meyer G, Vicaut E, Danays T, Agnelli G, Becattini C, BeyerWestendorf J, et al. Fibrinolysis for patients with intermediate-risk pulmonary embolism. N Engl J Med. 2014;370(15):1402-11. https://
doi.org/10.1056/NEJMoa1302097

3. Konstantinides SV, Vicaut E, Danays T, Becattini C, Bertoletti L, Beyer-Westendorf $\mathrm{J}$, et al. Impact of Thrombolytic Therapy on the Long-Term Outcome of Intermediate-Risk Pulmonary Embolism. J Am Coll Cardiol. 2017 Mar 28;69(12):1536-1544. https://doi. org/10.1016/j.jacc.2016.12.039

\footnotetext{
1. Unidade de Circulação Pulmonar, Disciplina de Pneumologia, Instituto do Coração, Hospital das Clínicas, Faculdade de Medicina, Universidade de São Paulo, São Paulo (SP) Brasil.

2. Instituto do Câncer, Hospital das Clínicas, Faculdade de Medicina, Universidade de São Paulo, São Paulo (SP) Brasil.

a. (iD http://orcid.org/0000-0002-4912-021X; b. iD http://orcid.org/0000-0003-0425-5548; c. (iD http://orcid.org/0000-0001-8943-1947;

d. (D) http://orcid.org/0000-0002-1385-5222; e. (D) http://orcid.org/0000-0002-0900-2737; f. (iD http://orcid.org/0000-0003-2789-9143
} 